\title{
FENOLOGÍA DEL BOSQUE DE LAS SUBREGIONES NATURALES: CHACO SEMIÁRIDO Y CHACO SERRANO, DE SANTIAGO DEL ESTERO, ARGENTINA
}

\section{FOREST PHENOLOGY OF SEMIARID-CHACO AND CHACO-SERRANO NATURAL SUB-REGIONS IN SANTIAGO DEL ESTERO, ARGENTINA}

\author{
José Luis Tiedemann ${ }^{1}$
}

\begin{abstract}
Resumen
El objetivo de este trabajo fue determinar los parámetros fenológicos del bosque nativo de las subregiones naturales: Chaco Semiárido y Chaco Serrano de la Provincia de Santiago del Estero, Argentina, mediante el software TIEMESAT y la serie temporal de NDVI VEGETATION SPOT. El periodo analizado (1998-2009) fue afectado por El Niño/La Niña, por cuanto fueron considerados dos periodos: húmedo (PH) y seco (PS). Se analizaron muestras del bosque Chaqueño semiárido; del bosque serrano de Guasayán y del bosque serrano de SumampaAmbargasta. El bosque Chaqueño semiárido y bosque serrano de Sumampa-Ambargasta tuvieron variaciones en el inicio de las estaciones de crecimiento entre periodos, siendo el más afectado el bosque de Sumampa-Ambargasta. En el PH el bosque Chaqueño semiárido tuvo los puntos medios en otoño, mientras que el bosque serrano de Guasayán y de Sumampa-Ambargasta los tuvieron en verano; en el PS las tres coberturas tuvieron los puntos medios en otoño; este desplazamiento estaría relacionado con las estratégicas adaptaciones para el uso eficiente del agua del estrato medio e inferior del bosque. El bosque Chaqueño semiárido tuvo, en ambos periodos, estaciones de crecimiento con similar producción total integrada, base y amplitud. El bosque serrano de Guasayán tuvo en el PS estaciones de crecimiento con mayor producción total integrada, por cuanto debería considerarse un efecto de saturación del NDVI en el PH. El bosque serrano de Sumampa-Ambargasta tuvo estaciones de crecimiento con similar producción total integrada en ambos periodos, sin embargo, las estaciones de crecimiento del PS tuvieron menor base, longitud y amplitud. La productividad de la vegetación estacionalmente activa de las tres coberturas no tuvo diferencias significativas entre periodos, esta respuesta estaría relacionada con la mayor biodiversidad, estratificación y por la presencia de especies leñosas de porte con raíces profundas que los conforman.
\end{abstract}

Palabras clave: NDVI; VEGETATION SPOT; TIMESAT; El Niño/La Niña; Bosque Chaqueño semiárido; Bosque Serrano; estación de crecimiento, periodo húmedo y seco.

Abstract
This paper aims at determining the phenological parameters of the native forest of the Semiarid-Chaco and Serrano-Chaco natural regions in the province of Santiago del Estero, Argentina, using TIMESAT software and the temporal series of NDVI VEGETATION SPOT. Since the study was carried out during a period (1998 to 2009) affected by ENSO, both wet and dry seasons were taken into account. Samples of the Semiarid Chaco forest, and Sierras de Guasayan and Sierras de Sumampa-Ambargasta mountain forests were analyzed. The Semiarid Chaco forest and Sierras de Sumampa-Ambargasta mountain forests showed variations at the beginning of the growing seasons between periods. Sumampa-Ambargasta mountain forests were the most affected. During the wet period, the Semiarid Chaco forest experienced its midpoint in autumn while Sierras de Guasayan and Sierras de Sumampa-Ambargasta mountain forests had theirs in summer. However, during the dry period the three areas underwent their midpoints in autumn. This displacement could be related to the strategic adaptations of the middle and lower forest strata to efficient water use. The Semiarid Chaco forest experienced, in both periods, growing seasons with similar fully integrated production, base and width. On the other hand, Sierras de Guasayan forest showed growing seasons with more fully integrated production in the dry season, a fact that could be the result of NDVI saturation effect during the wet period. Sumampa-Ambargasta mountain forests had similar growing seasons with fully integrated production in both periods. However, the growing season during the dry period had smaller base, length and width. The productivity of the seasonally active vegetation of the three areas showed no 
significant differences between the periods, which could be due to their greater biodiversity, stratification and the presence of woody species with deep roots.

Key words: NDVI, VEGETATION SPOT; TIMESAT; ENSO; Semiarid Chaco and Serrano Chaco; growing season; wet and dry period.

\section{Introducción.}

La fenología estudia los eventos periódicos del ciclo de vida de las plantas, como la floración, fructificación, brotación y senescencia de las hojas. Estos ciclos se encuentran fuertemente influenciados por la variación estacional de la precipitación y la temperatura (Reed et al., 1994; Cleland et al., 2007), por la composición de especies, estratificación y topografía (Fisher et al., 2006), por los cambios en las coberturas de la tierra (Heumann et al., 2007) y por el cambio climático global (Cleland et al., 2007) o incremento de la temperatura global (Linderholm, 2006).

Los sensores remotos fueron utilizados para observar cómo la fenología ha cambiado en las últimas décadas (Cleland et al., 2007). Los datos derivados de satélite proveyeron una manera eficiente para realizar el monitoreo de la fenología de la vegetación a escala regional y global (Kang et al., 2003). El índice de vegetación de diferencia normalizada - NDVI, es el índice estándar para estudios fenológicos, principalmente por su disponibilidad y por su fuerte herencia (Reed et al., 2003).

La disponibilidad de series temporales de datos derivados de satélites como el NOAA y Vegetation, con resolución temporal diaria y moderada resolución espacial de $1 \mathrm{~km}^{2}$ por pixel, fueron claves para el desarrollo de estudios fenológicos sobre grandes extensiones. Las series temporales posibilitaron la determinación de las fases de crecimiento activo de la vegetación (Reed et al., 2003) a escalas globales, continentales y regionales (Chen et al., 2004; Cleland et al., 2007). Reed et al. (1994) derivaron, a partir de series temporales de NDVI, parámetros fenológicos generales, como la entrada del verde, pico de máximo de NDVI, la tasa de crecimiento, la tasa de senescencia, fin del verde, y tiempo integrado NDVI de bosques y pasturas en USA. White et al. (1997) desarrollaron modelos fenológicos predictivos de ecosistemas específicos para ser aplicados a escala continental; mientras que Heumann et al. (2007) estimaron los cambios en la fenología de la vegetación de las regiones de Sahel, Sudan y Guinea, a partir de series temporales de NDVI, determinando significativas tendencias positivas en la longitud y en el fin de las estaciones de crecimientos de Sudan y Guinea, y sin tendencias positivas en el Sahel. Chen et al. (2000) mediante umbrales de frecuencias fenológicas acumulativas y su correspondiente verde, examinaron las relaciones estacionales entre la fenología de comunidades vegetales y el NDVI a escala regional en China.

De acuerdo al índice tendencias regionales de sequías (Minetti, 2009) el Noroeste Argentino, región en donde se encuentra la provincia de Santiago del Estero y en ella las áreas de muestreo de bosque, tuvo un periodo con excesos hídricos o periodo húmedo (PH) entre los años 1998 y 2004 y un periodo seco (PS) entre los años 2004 y 2009. La variación en los patrones de precipitaciones regional estaría fuertemente asociada a la influencia del ENSO (FAS, 2009). Este fenómeno, tele-detectado en la década del 90’ (Anyamba \& Eastman, 1996) fue asociado con dramáticos cambios en los patrones de precipitaciones a diversas escalas, resultando en precipitaciones por encima de lo normal en algunas regiones y por debajo de lo normal en otras (Anyamba et al., 2002; Barbosa et al, 2006). El ENSO es un indicador clave de variabilidad climática interanual a escala global, según Anyamba et al. (2002) la transición entre estas fases es generalmente inversa. De acuerdo a Linderholm (2006) y Bradley et al. (2007) en ecosistemas semiáridos, como Santiago del Estero, la fenología es mucho más sensible a la precipitación y evapotranspiración que a la temperatura y foto período. Adicionalmente, en áreas donde los patrones de precipitación fueron fuertemente influenciados por el ENSO debido al cambio climático global, es esperable que la frecuencia e intensidad del evento ENSO en esas áreas se incremente (Cleland et al., 2007). La sequía afecta severamente la fenología de la vegetación natural (Karnieli et al., 2002). Por cuanto, los objetivos de este trabajo fueron determinar los parámetros fenológicos del bosque de las subregiones naturales Chaco Semiárido y Chaco Serrano de la Provincia de Santiago del Estero, Argentina, y cuantificar su respuesta ante la influencia de dos periodos: húmedo (1998-2004) y seco (2004-2009).

\section{Materiales y métodos.}

La provincia de Santiago del Estero se localiza entre $25^{\circ} 40^{\prime}$ y $30^{\circ} 30^{\prime}$ de latitud Sur y entre $61^{\circ} 40^{\prime}$ y $65^{\circ} 10^{\prime}$ de longitud Oeste (Figura 1), posee una superficie de $145.758 \mathrm{~km}^{2}$ (Torres Bruchman, 1981). El tipo climático es semiárido $B S h w$ según la clasificación climática de Köppen, la evapotranspiración anual oscila entre los 900-1.100 $\mathrm{mm}$, con déficit hídrico todo el año, la isoterma de los $47^{\circ} \mathrm{C}$ cubre la provincia, la media anual histórica es de $27,7^{\circ} \mathrm{C}$, y la precipitación media es de $550 \mathrm{~mm}$ (Torres Bruchman, 1981). 

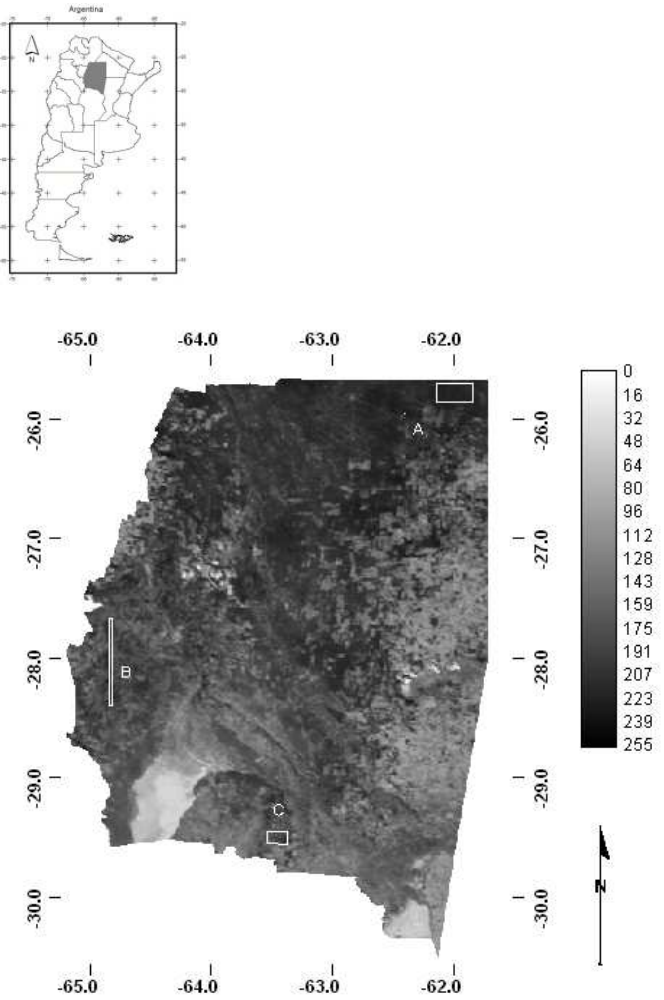

Figura 1. Mapa de la Provincia de Santiago del Estero en Argentina, y la ubicación espacial de las áreas de muestreo: (A) Bosque Chaqueño semiárido; (B) Bosque Chaqueño Serrano de las Sierras de Guasayán y (C) Bosque Chaqueño Serrano de las Sierras de Sumampa y Ambargasta.

De acuerdo al estudio integral de la Región del Parque Chaqueño (1999), la subregión Chaco semiárido se extiende por casi todo el territorio de Santiago del Estero, excepto una pequeña parte del sudeste perteneciente al distrito Chaco húmedo. A su vez, la subregión Chaco Serrano, formada por serranías del grupo de las sierras Pampeanas y las áreas más bajas de las sierras Subandinas, se extiende al sur de Santiago del Estero por las sierras de Sumampa y Ambargasta, y al sudoeste por las sierras de Guasayán (SAyDS, 2007). El bosque Chaqueño semiárido (BCHS) se caracteriza por ser xerofítico y abierto y es considerado la vegetación clímax de la región (SAyDS, 2007). Estructuralmente, está conformado por cuatro estratos bien definidos, el estrato superior conformado por especies leñosas de porte como el quebracho colorado Santiagueño (Schinopsis lorentzii) y el quebracho blanco (Aspidosperma quebracho-blanco); el estrato medio conformado por los géneros Zizyphus, Cercidium, Celtis, Acanthosyris, Jordania, Caesalpinea. Entre las leguminosas principales de porte leñoso se encuentran los géneros Prosopis, Mimozyganthus, Geoffroea, Cercidium, Caesalpinea; el estrato arbustivo conformado por los géneros Acacia, Mimosa, Geoffroea, Prosopis, Atamisquea, entre otras; y el estrato herbáceo conformado por gramíneas y Dicotiledóneas (SAyDS, 2007). En el bosque Serrano de las sierras de Guasayán (BSG), hay un predominio, de árboles de gran porte en el estrato superior (SAyDS, 2007) y de arbustos y herbáceas en el estrato inferior, con características similares al bosque Chaqueño Semiárido (SAyDS, 2004). La especie arbórea característica es el horco quebracho (Schinopsis haenkeana), asociado al molle de beber (Lithaea ternifolia) (SAyDS, 2007). Se destaca, que este bosque, es enriquecido en el norte por elementos florísticos de la selva Tucumano-Oranense (Zerda \& Moreira, 2006; SAyDS, 2007) como Piptadenia macrocarpa y $P$. excelsa, entre otras especies (Sarmiento, 1963). Las sierras de Sumampa y Ambargasta, son la continuación hacia el norte de las Sierras Pampeanas de la Provincia de Córdoba (Angueira et al., 2007; SAyDS, 2007). De acuerdo a Giménez \& Hernández (2008), en el bosque maduro de las Sierras de Sumampa y Ambargasta (BSSA), predomina el Quebracho colorado (Schinopsis lorentzii) con más de $40 \mathrm{~cm}$ de diámetro a $1,3 \mathrm{~m}$ de altura, asociado con Quebracho blanco (Aspidosperma quebracho blanco), Mistol (Ziziphus mistol) y en menor grado especies del género Prosopis alba y nigra. El estrato arbustivo representado por especies como Celtis pallida, Prosopis elata, Prosopis sericantha, Acacia aroma, Mimosa detinens, Ximenia americana, entre otras.

Con el fin de captar, específicamente la fenología del bosque Chaqueño, las áreas de muestreo debían cumplir con dos requisitos: 1.- que el $100 \%$ de la superficie del área de muestreo debe estar cubierta por bosque Chaqueño y 2.- que dicha superficie de cobertura vegetal debía mantenerse durante todo el periodo analizado; en base a esto, se delimitaron tres áreas de muestreo: Muestra A: abarca $561 \mathrm{~km}^{2} \mathrm{de}$ BCHS (Fig. 1 A); Muestra B: abarca $213 \mathrm{~km}^{2} \mathrm{de}$ BSG (Fig. 1 B); y la Muestra C: abarca $205 \mathrm{~km}^{2} \mathrm{de}$ BSSA (Fig. $1 \mathrm{C}$ ).

La variable utilizada es el índice de vegetación de diferencia normalizada (NDVI). El NDVI es la combinación aritmética de las porciones roja $\left(R E D^{\lambda}\right.$ $0,58-0,68 \mu \mathrm{m})$ e infrarroja cercano $\left(N I R^{\lambda 0^{0,725-1,1} \mu \mathrm{m}}\right) \mathrm{del}$ espectro y se obtiene de la siguiente ecuación: NDVI $=(\mathrm{NIR}-\mathrm{RED}) /(\mathrm{NIR}+\mathrm{RED}) \quad($ Tucker 1979$) . \quad \mathrm{Se}$ derivaron 387 mapas originales de NDVI de Sudamérica, estandarizados a 8 bits, sensor remoto Vegetation SPOT, desarrollados por el Centro Nacional de Estudios Espaciales, Francia. Los mapas de NDVI poseen una resolución espacial de $1 \mathrm{~km}^{2}$ y una resolución temporal síntesis diez días en base al criterio NDVI Máximo Valor Compuesto al límite de la atmósfera (Passot, 2000).

Los parámetros fenológicos del bosque Chaqueño Semiárido y Serrano fueron extractados de la serie 
temporal NDVI VEGETATION mediante el software TIMESAT. El TIMESAT fue desarrollado por Jönson \& Eklundh (2004), exclusivamente, para suavizar y extractar, parámetros fenológicos de series temporales derivadas de sensores remotos. El TIMESAT posibilita implementar un método basado en el ajuste por mínimos cuadrados, conocido como adaptativo filtro Savitzky-Golay (Jönson \& Eklundh, 2004). El adaptativo filtro Savitzky-Golay es un método simple y robusto, que posee una elevada eficiencia en la reducción de contaminación y del ruido global de series temporales de NDVI (Savitzky \& Golay, 1964, citado por Chen et al., 2004; Hird \& McDermid, 2009).

Para determinar el efecto de la variación en los patrones de precipitación sobre los parámetros fenológicos, la serie temporal NDVI VEGETATION SPOT, fue dividida en dos: 1.- la serie temporal del periodo húmedo $(\mathrm{PH})$, conformada por 154 mapas de NDVI, que se inicia el 01/06/1998 y finaliza el $01 / 09 / 2004$, esta serie temporal contiene a seis estaciones unimodales de crecimiento (98-99; 99-00; 00-01; 01-02; 02-03; 03-04); y 2.- la serie temporal del periodo seco (PS), conformada por 175 mapas de NDVI, que se inicia el 21/05/2004 y finaliza el 21/11/2009; esta serie temporal contiene a cinco estaciones unimodales de crecimiento (04-05; 05-06; 06-07; 07-08; 08-09).

Los parámetros operativos utilizados para el análisis de las series temporales en TIMESAT fueron los siguientes: amplitud 10; número de estaciones 0,5; $\mathrm{N}^{\mathrm{o}}$ interacciones 3; fuerza de adaptación 2; método generador de parámetros estacionales filtro SavitzkyGolay; window Savitzky-Golay 2.4.4; umbral $20 \%$.

Parámetros fenológicos a extractar (Fig. 2):

Inicio de estación de crecimiento (IEC): es el punto en el tiempo de la función de ajuste que ha incrementado su valor en un $20 \%$, con respecto a la distancia que existe entre el punto mínimo izquierdo y el punto máximo de la curva ajustada (Jönson \& Eklundh, 2006). Se adoptó el umbral del 20\%, como una variación aceptable del valor de NDVI para el tiempo $t$, (Jönson \& Eklundh, 2002; Jönson \& Eklundh, 2006, Delbart et al., 2006; Heumann et al., 2007). Fin de estación de crecimiento (FEC): es el punto mínimo derecho de la curva de ajuste, se define de manera similar que el punto mínimo izquierdo o IEC. Punto medio (PM): es el punto en el tiempo de la función de ajuste que se encuentra en el medio de los puntos temporales izquierdo y derecho de la función de ajuste que han incrementado en un $90 \%$ con respecto al mínimo derecho e izquierdo respectivamente. Longitud (LEC): está dada por la diferencia en tiempo del último y primer mapa de NDVI S10 incluidos entre los puntos IEC y FEC. Amplitud (AEC): es la diferencia entre el valor del NDVI máximo (PM) y la base. Base (BEC): es la media entre el NDVI mínimo izquierdo (IEC) y el
NDVI mínimo derecho (FEC). Primer integral (h): es la superficie comprendida entre la función de ajuste y el nivel medio de los mínimos izquierdo (IEC) y derecho (FEC). Esta integral representa la productividad integrada de la vegetación fotosintéticamente activa (PVFA) de los bosques. Mediante la prueba $\mathrm{T}$ para muestras independientes, se realizó la comparación de medias de la PVFA de los bosques, siendo el criterio clasificatorio el PH y PS (Infostat, 2004). Segunda integral (i): es la superficie comprendida entre la función de ajuste y el nivel cero, y representa la producción total integrada (PTI) de los bosques. Mediante la prueba $\mathrm{T}$ para muestras independientes, se realizó la comparación de medias de la PTI de los bosques, siendo el criterio clasificatorio el PH y PS (Infostat, 2004).

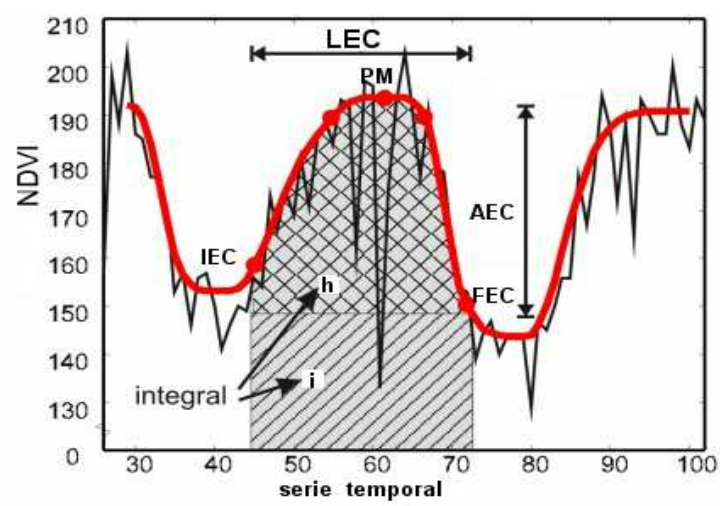

Figura 2. Parámetros fenológicos de la vegetación extractados mediante TIMESAT - inicio (IEC) y fin (FEC) de la estación de crecimiento (EC), punto medio (PM); longitud (LEC); Amplitud (AEC); $1^{\mathrm{a}}$ integral (h); $2^{\mathrm{a}}$ integral (i) (fuente: Jönson \& Eklundh, 2002).

\section{Resultados.}

La serie temporal de NDVI y los parámetros estacionales extractados mediante TIMESAT del bosque Chaqueño Semiárido (BCHS) se presentan, respectivamente, en la Figura 3 y en Tabla 1.

Fenología del BCHS: el inicio de la estación de crecimiento en el PH fue el 01 de octubre y el fin fue el 01 de agosto; mientras que el inicio de la estación de crecimiento en el PS fue el 21 de octubre y el fin fue el 21 de agosto (Tabla 1). El punto medio en el PH fue el 11 de marzo, en el PS el punto medio fue el 01 de marzo. El NDVI máximo fue similar en el $\mathrm{PH}$ y PS, sin embargo, los desvíos fueron mayores en el PH (Tabla 1). La base y la amplitud de las estaciones de crecimiento fueron similares en ambos periodos, sin embargo, los desvíos de ambos parámetros fueron superiores en el PH (Tabla 1). No se encontraron diferencias significativas $(p>0.05)$ entre las respectivas, productividad de la vegetación estacionalmente activa y la producción total integrada entre periodos (Tabla 1). 
Tabla 1. Parámetros estacionales extractados mediante TIMESAT. BCHS= bosque Chaqueño Semiárido; $B S G=$ bosque Serrano de las Sierras de Guasayán; BSSA= bosque Serrano de las Sierras de Sumampa-Ambargasta; $\mathrm{PH}=$ periodo húmedo; $\mathrm{PS}=$ periodo seco; IEC y $\mathrm{FEC}=$ inicio y fin de la estación de crecimiento; $\mathrm{PM}=$ punto medio; NDVI máx. = NDVI máximo; $\mathrm{DS}=$ desvío estándar; $\mathrm{BEC}=$ base de la estación de crecimiento; $\mathrm{AEC}=$ amplitud de la estación de crecimiento; PTI= producción total integrada; PVFA= productividad de la vegetación estacionalmente activa. $(*)=$ diferencias significativas entre periodos $(p<0,01)$.

\begin{tabular}{|c|c|c|c|c|c|c|c|c|c|c|c|c|}
\hline & & IEC & PM & FEC & $\begin{array}{l}\text { NDVI } \\
\text { Máx. }\end{array}$ & $\begin{array}{c}\text { DS } \\
\text { NDVI } \\
\text { Máx. }\end{array}$ & BEC & $\begin{array}{c}\text { DS } \\
\text { BEC }\end{array}$ & AEC & $\begin{array}{c}\mathrm{DS} \\
\mathrm{AEC}\end{array}$ & PTI & PVFA \\
\hline \multirow{3}{*}{ PH } & BCHS & 01-oct & 11-mar & 01-ago & 236 & 10 & 160 & 19 & 75 & 13 & 4485 & 1032 \\
\hline & BSG & 21-oct & 21-ene & 21-jul & 223 & 3 & 132 & 8 & 91 & 7 & $4002 *$ & 1261 \\
\hline & BSSA & 01-oct & 21-feb & 01-ago & 231 & 5 & 169 & 5 & 80 & 11 & 4533 & 865 \\
\hline \multirow{3}{*}{ PS } & BCHS & 21-oct & 01-mar & 21-ago & 236 & 6 & 160 & 10 & 75 & 9 & 5154 & 1186 \\
\hline & BSG & 21-oct & 01-mar & 01-ago & 227 & 8 & 130 & 4 & 97 & 11 & $4702 *$ & 1557 \\
\hline & BSSA & 11-nov & 21-mar & 11-ago & 228 & 12 & 148 & 16 & 62 & 6 & 4497 & 1156 \\
\hline
\end{tabular}
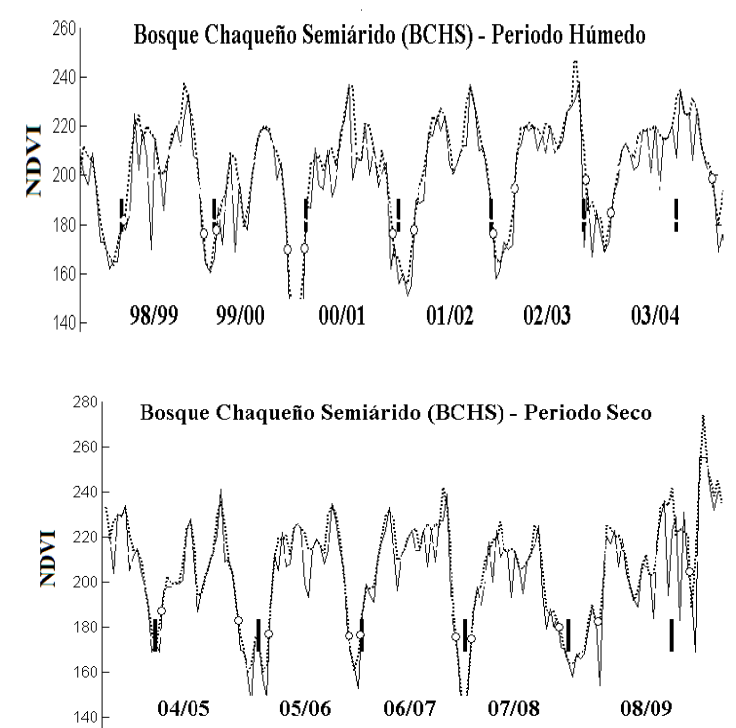

Figura 3. Serie temporal del NDVI del bosque Chaqueño semiárido (BCHS) en el periodo húmedo (izq) y seco (der); siendo (o ) = IEC y FEC; $(-)=$ serie temporal original de NDVI VGT; $(. . . .)=$. nueva serie temporal ajustada con el adaptativo filtro Savitzky-Golay, y ( 1$)$ = límite entre estaciones de crecimientos.

La serie temporal de NDVI y los parámetros estacionales extractados mediante TIMESAT del bosque serrano de Guasayán (BSG) se presentan, respectivamente, en la Figura 4 y en la Tabla 1.

Fenología del BSG: el inicio de la estación de crecimiento en el PH y PS fue el 21 de octubre, el fin en el PH fue el 21 de julio, mientras que en el PS fue el 01 de agosto (Tabla 1). El punto medio del PH fue el 21 de enero y en el PS el 01 de marzo (Tabla 1). El NDVI máximo en el $\mathrm{PH}$ fue ligeramente inferior pero con menores desvíos que en el PS (Tabla 1). Las bases de las estaciones de crecimiento de ambos periodos fueron similares, sin embargo, los desvíos fueron menores en el PS (Tabla 1). La amplitud de la estación de crecimiento y los desvíos fueron mayores en el PS (Tabla 1). Se encontraron diferencias significativas $(p<0.01)$ entre la producción total integrada entre periodos (Tabla 1); a su vez, no se encontraron diferencias significativas $(p>0.05)$ entre la productividad de la vegetación estacionalmente activa entre periodos (Tabla 1).

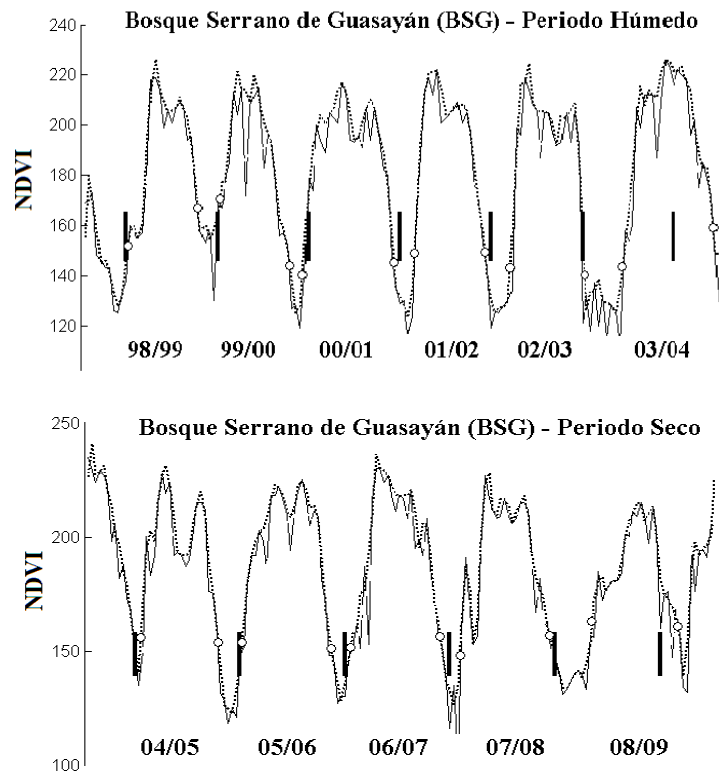

Figura 4. Serie temporal del NDVI del bosque Chaqueño Serrano de Guasayán (BSG) en el periodo húmedo (izq) y seco (der); siendo (o) = IEC y FEC; $(-)=$ serie temporal original NDVI VGT $;(. . . .$.$) = nueva serie temporal ajustada con el$ adaptativo filtro Savitzky-Golay y ( । ) = límite entre estaciones de crecimientos. 
La serie temporal de NDVI y los parámetros estacionales extractados mediante TIMESAT del bosque serrano de Sumampa-Ambargasta (BSSA) se presentan, respectivamente, en la Figura 5 y en la Tabla 1.

Fenología del BSSA: durante el PH, el inicio de la estación de crecimiento fue el 01 de octubre y el fin fue el 01 de agosto (Tabla 1). Durante el PS, el inicio de la estación de crecimiento fue el 11 de noviembre y el fin fue el 11 de agosto. El punto medio en el PH fue el 21 de febrero, y en el PS y el 21 de marzo (Tabla 1). El NDVI máximo del PH fue ligeramente superior al del PS, sin embargo, en el PS tuvo gran variabilidad (Tabla 1). Las bases de la estación de crecimiento fueron mayores en el $\mathrm{PH}$ y con bajos desvíos, por el contrario, los desvíos de las bases de las estaciones de crecimiento en el PS fueron elevados (Tabla 1). La amplitud de las estaciones de crecimiento en el $\mathrm{PH}$ fueron mayores y con mayor variabilidad que las del PS (Tabla 1). No se encontraron diferencias significativas $(p>0.05)$ entre las respectivas, producción total integrada y productividad de la vegetación estacionalmente activa entre periodos (Tabla 1).
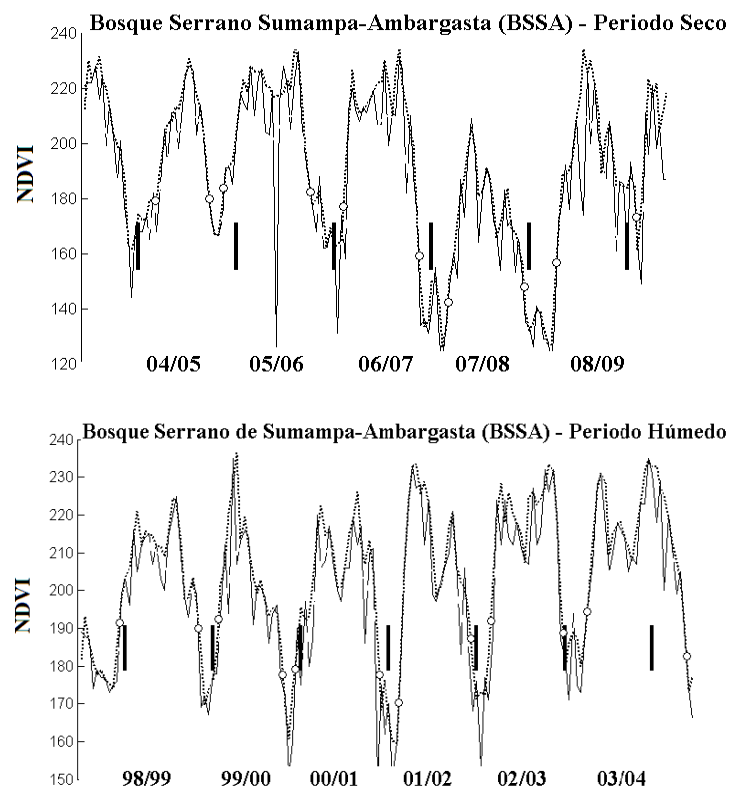

Figura 5. Serie temporal NDVI del bosque Chaqueño Serrano de Sumampa-Ambargasta (BSSA) en el periodo húmedo (izq) y seco (der); siendo $(\mathrm{O})=$ IEC y FEC $;(-)=$ original serie de tiempo NDVI VGT; $(. . . .$.$) = nueva serie temporal$ ajustada con el adaptativo filtro Savitzky-Golay y ( । ) = límite entre estaciones de crecimientos.

\section{Discusiones.}

El bosque Chaqueño semiárido tuvo un desplazamiento de 20 días en el inicio y fin de las estaciones de crecimiento del PS con respecto al PH; sin embargo, se destaca que esta cobertura mantuvo estaciones de crecimiento de similar longitud entre periodos. Delbart et al. (2005) determinaron variaciones de \pm 15 días en el inicio de las estaciones de crecimiento en Siberia central mediante NDWI derivado de VEGETATION SPOT; a su vez, Delbart et al. (2006) determinaron variaciones de \pm 19 días en el inicio de las estaciones de crecimiento en el periodo 1982-2004 a partir de NDVI y NDWI derivados de AVHRR-NOAA y VEGETATION SPOT, en ecosistemas del Norte de Eurasia. El bosque de sierras de Guasayán no tuvo variación en el inicio de la estación de crecimiento, si tuvo un mínimo desplazamiento en el fin de las estaciones de crecimiento entre periodos. El inicio de las estaciones de crecimiento del bosque de sierras de SumampaAmbargasta en el PS tuvo un retraso de 40 días con respecto al $\mathrm{PH}$, esta cobertura fue la más afectada por la sequía regional. De acuerdo Bradley et al. (2007) el inicio y fin de las estaciones de crecimiento son fuertemente afectados, en corto término, por fluctuaciones en las precipitaciones. El desplazamiento o retraso en el inicio y fin de las estaciones de crecimiento del bosque Chaqueño semiárido y el de sierras de Sumampa-Ambargasta en el PS, estaría relacionado, con la diversidad de especies que conforman el estrato inferior y medio de las mismas. Estos estratos responden de manera diferenciada ante la disponibilidad o no de humedad (Wang et al., 2003). De acuerdo a Doktor et al. (2009) la diferencia temporal en la respuesta del verde del estrato inferior con respecto al estrato superior es generalmente de 15 días, sin embargo, se destaca la respuesta, altamente diferenciada de las especies que conforman el bosque de sierras de SumampaAmbargasta. La variación temporal en el inicio y fin de las estaciones de crecimiento, del bosque Chaqueño semiárido y el bosque serrano de Guasayán podría considerarse como mínimas, sobre todo si comparamos resultados obtenidos por Chen et al. (2000) quienes al explorar las relaciones entre fenología de las plantas y mediciones de verde derivados de satélite de tres sitios en China con diferencias en clima y vegetación, determinaron que la longitud media de las estaciones de crecimiento varia de 188 días en el Norte y de 259 días en el Sur del área en estudio. Así mismo, es importante destacar que el proceso de síntesis NDVI diez días Máximo Valor Compuesto, tiene gran incidencia en la fecha de ocurrencia del inicio y fin de las estaciones de crecimiento. En este sentido, Brown \& de Beurs (2008) vieron significativamente disminuida la determinación del inicio de las estaciones de crecimiento de cultivos anuales en el Oeste de África, en el proceso de síntesis de NDVI diez días MVC del sensor VEGETATION SPOT por la elevada contaminación residual de nubes. 
Los puntos medios del bosque serrano de Guasayán y de Sumampa-Ambargasta en el PH fueron en verano (enero y febrero), desplazándose en el PS al otoño (marzo). La ocurrencia temporal de los puntos medios de estas coberturas en ambos periodos es consistente; durante el verano de las estaciones de crecimiento del $\mathrm{PH}$, las comunidades vegetales del bosque serrano de Guasayán y el de SumampaAmbargasta no serían afectadas por el estrés térmico e hídrico debido a la elevada disponibilidad de humedad edáfica, por el contrario, en el verano del PS, el desplazamiento del los puntos medios al otoño, estaría fuertemente relacionado con las estrategias de las especies que conforman los estratos inferiores para el uso eficiente del agua ante la escasez de disponibilidad de humedad edáfica. Similar estrategia utilizarían las comunidades vegetales que conforman el bosque Chaqueño semiárido debido a que los puntos medios de esta cobertura ocurrieron, en ambos periodos, en otoño (marzo).

Se destaca que no se encontraron diferencias significativas $(p>0.05)$ en la productividad de la vegetación estacionalmente activa del bosque Chaqueño semiárido, bosque serrano de Guasayán y de Sumampa-Ambargasta entre periodos, esta respuesta estaría fuertemente relacionada con la elevada biodiversidad, estratificación y a las especies leñosas de porte con raíces profundas que los conforman.

El bosque Chaqueño semiárido, no tuvo diferencias significativas en la producción total integrada entre periodos, este parámetro se correspondió con similar NDVI máximo, base y amplitud. Estos resultados difieren de los obtenidos por Heumann et al. (2007) quienes al analizar los cambios producidos en la fenología de la vegetación en el Sahel, Sudán y Guinea a partir de series temporales de NDVI (AVHRR/NOAA) mediante TIMESAT, determinaron, que los incrementos en la producción total integrada del NDVI en zonas con menor disponibilidad de humedad (Shael) se correspondían con incrementos en la amplitud de las estaciones de crecimiento, mientras que en zonas con mayor disponibilidad de humedad (Sudán y Guinea) se correspondían con incrementos en la longitud y decrementos en la amplitud de las estaciones de crecimiento.

El bosque serrano de Guasayán tuvo diferencias significativas en la producción total integrada entre periodos, esta cobertura tuvo una mayor producción total integrada durante el PS; esta respuesta estaría relacionada con un efecto de saturación del NDVI. En este sentido, Jönson \& Eklundh (2004) determinaron, en áreas con coberturas de especies siempre verdes, que la productividad de la vegetación estacionalmente activa es generalmente pequeña, incluso aún, cuando la producción total integrada es elevada. El NDVI es ampliamente conocido por su saturación ante follajes densos (Anderson et al., 2007). La saturación del NDVI se produce debido al incremento en la densidad de hojas verdes (efecto de la disponibilidad de humedad edáfica del $\mathrm{PH}$ ), por cuanto la actividad fotosintética de las hojas inferiores disminuye progresivamente al recibir cada vez menos radiación fotosintéticamente activa (RFA), al llegar al punto de saturación la absorción de la RFA se hace constante y por ende el NDVI (Nicholson et al., 1990). Xiao et al. (2006) al cuantificar la variabilidad espacial y temporal de la fenología de hojas en bosques tropicales de Sudamérica, determinaron efectos de saturación del NDVI debido a los frecuentes elevados valores de índice de área foliar (IAF) $>4 \mathrm{~m}^{2} / \mathrm{m}^{2}$, para este tipo de coberturas. A su vez, Doktor et al. (2009) al explicar las diferencias existentes entre el inicio de la estación de crecimiento derivado de satélites y observaciones de tierra de paisajes en Europa central, determinaron que la saturación del NDVI se produce en áreas forestales con IAF $>3 \mathrm{~m}^{2} / \mathrm{m}^{2}$.

El bosque serrano de Sumampa-Ambargasta, no tuvo diferencias en la producción total integrada entre periodos, sin embargo, las estaciones de crecimiento en el PS tuvieron menores bases, longitud y amplitud; los resultados son consistentes y coinciden con los de Heumann et al. (2007) quienes determinaron que en zonas húmedas (Sudan o Guine) las menores amplitudes indicarían una mejor condición de crecimiento de la vegetación. Adicionalmente y de acuerdo a Delbart et al. (2006) la variación en la disponibilidad de humedad incide indirectamente sobre la amplitud.

\section{Conclusiones.}

El software TIMESAT posibilitó extractar los parámetros fenológicos a partir de la serie temporal de NDVI VEGETATION SPOT, del bosque Chaqueño semiárido y del bosque serrano de sierras de Guasayán y de Sumampa-Ambargasta de Santiago del Estero.

Los parámetros fenológicos del bosque Chaqueño semiárido, del bosque serrano de Guasayán y de Sumampa-Ambargasta fueron afectados por los periodos. La respuesta diferenciada entre periodos de las tres coberturas estaría relacionada con las estratégicas adaptaciones, morfológicas, fisiológicas y de comportamiento para el uso eficiente del agua de los estratos inferiores y medios del bosque. Se destaca que la productividad de la vegetación estacionalmente activa de las tres coberturas no fue afectada entre periodos, esta respuesta estaría fuertemente relacionada con la elevada biodiversidad, estratificación y a la presencia de especies leñosas de porte con raíces profundas que los conforman.

\section{Agradecimientos.}

- Al Dr. Hugo R. Zerda, director de los proyectos CICyT - UNSE: "Monitoreo de quemas e incendios y su relación con la dinámica de la 
vegetación en la provincia de Santiago del Estero $\left(\mathrm{N}^{\circ}\right.$ 23/B081) y "Monitoreo satelital de la distribución espacial y temporal de las coberturas y usos de la tierra de la provincia de Santiago del Estero" ( $\mathrm{N}^{\circ}$ 23/B060) en los cuales se enmarcaron la investigación de este trabajo.

- A la Dra. Liliana Diodato, directora del Instituto de Protección Vegetal de la Facultad de Ciencias Forestales, UNSE, por aportar el marco institucional para el desarrollo de las investigaciones.

\section{Literatura citada.}

Angueira C; Prieto D; Lopez J \& Barraza G. 2007. Geomorfología. En: Sistemas de Información Geográfica de Santiago del Estero, CD ROOM, ISBN 987- 521-170-2. Instituto de Tecnología Agropecuaria, EEA Santiago del Estero.

Anderson O.L; Malhi Y; Shimabukro \& Argao L.E. 2007. Evaluating MODIS vegetation and water indices for detecting canopy stress during the 2005 drought in Amazonia. Anais XIII Simpósio Brasileiro de Sensoriamento Remoto, Florianópolis, Brasil, 21-26 abril 2007, INPE, p. 6593-6595.

Anyamba A. \& Eastman J.R. 1996. Interannual variability of NDVI over Africa and its relation to EI Nino/Southern Oscillation. Int. J. Remote Sensing, 13:2533-2548.

Anyamba A; Tucker J.C \& Mahoney R. 2002. From El Niño to La Niña: Vegetation Response Patterns over East and Southern Africa during the 1997-2000 Period. Journal of climate 15:3096-3103.

Barbosa H.A; Huete A.R \& Baethgen W.E. 2006. A 20-year study of NDVI variability over the Northeast Region of Brazil. Journal of Arid Environments 67:288-307.

Bradley B.A; Jacob R.W; Hermance J.F \& Mustard J.F. 2007. A curve fitting procedure to derive inter-annual phenologies from time series of noisy satellite NDVI data. Remote Sensing of Environment 106:137-145.

Brown M.E \& de Beurs K.M. 2008. Evaluation of multisensor semi-arid crop season parameters based NDVI and rainfall. Remote Sensing of Environment 112:2261-2271.

Chen X; Tan Z; Schwartz M.D \& Xu C.H. 2000. Determining the growing season of land vegetation on the basis of plant phenology and satellite data in Northern China. Int. J. Biometeopapel, 44:97-101.

Chen J; Jonson P; M Tamura; Gu Z; Matsushita B \& Elkmundh L. 2004. A simple method for reconstructing a high-quality NDVI time-series data set based on the Savitzky-Golay filter. Remote Sensing of Environment 91:332-344.

Cleland E; Chuine I; Menzel A; Mooney H.A \& Schwartz M.D. 2007. Shifting plant phenology in response to global change. TRENDS in Ecology and Evolution 22:357-364.

Delbart N; Kergoat L; Le Toan T; L'Hermitte J \& Picard G. 2005. Determination of phenological dates in boreal regions using normalised difference water index. Remote Sensing of Environment, 97:26-38.

Delbart N; Le Toan T; Kergoat L \& Fedotova V. 2006. Remote sensing of spring phenology in boreal regions: A free of snow-effect method using NOAA-
AVHRR and SPOT-VGT data (1982-2004). Remote Sensing of Environment 101:52-62.

Doktor D; Bondeau A; Koslowski D \& Badeck F. 2009. Influence of heterogeneous landscapes on computed green-up dates based on daily AVHRR NDVI observations. Remote Sensing of Environment 113:2618-2632

Estudio Integral de la Región del Parque Chaqueño.1999. Proyecto Bosque Nativos y Áreas Protegidas. Préstamo BIRF No 4084-AR. Gerencia Técnica Bosque Nativos, Dirección de Bosques, Secretaria de Recursos Naturales y Desarrollo Sustentable.

Fisher JI; Mustard J.F \& Vadeboncoeur M.A. 2006. Green leaf phenology at Landsat resolution: Scaling from the field to the satellite. Remote Sensing Environment 100:265-279.

Giménez A. M. \& Hernández P. 2008. Vegetación del Chaco Semiárido Provincia de Santiago del Estero. En: Biodiversidad en ambientes naturales del Chaco Argentino. Facultad de Ciencias Forestales, UNSE, Ed. Lucrecia, 87 pp.

Heumann B.W; Seaquist J.W; Eklundh L \& Jonsson P. 2007. AVHRR derived phenological change in the Sahel and Soudan, Africa, 1982-2005. Remote Sensing of Environment 108:385-392.

Hird J.N \& McDermid G.J. 2009. Noise reduction of NDVI time series: An empirical comparison of selected techniques. Remote Sensing of Environment 113:248-258.

InfoStat. 2004. InfoStat, versión 2004. Manual del Usuario. Grupo InfoStat, FCA, Universidad Nacional de Córdoba. $1^{\text {a }}$ Edición, Editorial Brujas, Argentina.

Jönson P. \& Eklundh L. 2002. Seasonality Extraction by Function fitting to Time- Series of satellite Sensor Data. IEEE Transactions on Geoscience and Remote Sensing, 40:1824-832.

.2004. TIMESAT - a program for analyzing timeseries of satellite sensor data. Computers \& Geosciences 30:833-845.

.2006. TimesatGUI. Users guide for version TIMESAT 2.2, $17 \mathrm{pp}$.

Kang S; Running W.S; Lim J; Zhao M; Park C \& Loehman R. 2003. A regional phenology model for detecting onset of greenness in temperate mixed forests, Korea: an application of MODIS leaf area index. Remote

Sensing of Environment 86:232-242.

Karnieli A; Gabai A; Ichoku C; Zaady E \& Shachak M. 2002. Temporal dynamics of soil and vegetation spectral responses in a semi-arid environment. International J. of Remote Sensing, 19:4073-4087.

Linderholm H.W. 2006. Growing season changes in the last century. Agricultural and Forest Meteorology 137:114.

Minetti L.J. 2009. Tendencia regional de sequías del Noroeste Argentino, Disponible http://www.labclisud.com.ar/Portada_indice.asp. Acceso: 11.07.2009.

Nicholson S.E; Davenport M \& Malo A.R. 1990. A comparison of the vegetation response to rainfall in the Shael and East Africa, using Normalized Difference Vegetation Index from NOAA AVHRR, Climatic Change 17:209-241.

Passot X. 2000. Vegetation Image processing method in the CTIV. In:VEGETATION 2000 PROCEEDINGS. Ed. 
Gilbert Saint, VEGETATION progamme Scientist; Centre National Détudes Spatiales. Lake Maggiore, Italy.

Reed B.C; Brown J.F; VanderZee D; Loveland T.R; Merchant J.W \& Ohlen D.O. 1994. Variability of land cover phenology in the USA. J Veg Sci, 5:703- 714.

Reed B.C; White M \& Brown J.F. 2003. Remote Sensing Phenology. En: Phenology: An Integrative Enviromental Science. Schwartz M. D. (Ed), Kluwer Academic Publisher Netherlands, 557 pp.

Sarmiento G. 1963. Las comunidades vegetales del Chaco Santiagueño. Tesis Doctoral. Universidad de Buenos Aires, Buenos Aires, Argentina, 104 pp.

Savitzky A \& Golay M.J.E. 1964. Smoothing and differentiation of data by simplified least squares procedures. Analytical Chemistry, 36:1627-1639.

Secretaria de Ambiente y Desarrollo Sustentable de la Nación. 2004. Parque Chaqueño. En: Atlas de los Bosques Nativos Argentinos, Proyecto Bosques Nativos y Áreas protegidas BIRF 4085-AR, Argentina, 244 $\mathrm{pp}$

Secretaria de Ambiente y Desarrollo Sustentable de la Nación. 2007. Informe

Regional Parque Chaqueño. En: Primer inventario nacional de bosques nativos, Proyecto Bosques Nativos y Áreas protegidas BIRF 4085-AR, Argentina, 114p.
Torres Bruchmann E. 1981. Climatología General y Agrícola de la Provincia de Santiago del Estero. Universidad Nacional de Tucumán, Tucumán, Argentina, $199 \mathrm{pp}$.

Tucker J.C. 1979. Red and Photographic infrared lineal combinations for monitoring vegetation, Remote Sensing of Environment, 8:127-150.

Wang J., Price K. P., \& Rich P. M. 2003. Temporal responses of NDVI to precipitation and temperature in the central Great Plains, USA. Int. J. Remote Sensing, 11:2345-2364.

White A.M; Thornton E.P \& Running W.S. 1997. A continental phenology model for monitoring vegetation responses to interannual climatic variability.

Global Biogeochemical Cycles, 2:217-234.

Xiao X; Hagen S; Zhang Q; Keller M \& Moore B III. 2006. Detecting leaf phenology of seasonally moist tropical forests in South America with multi-temporal MODIS images. Remote Sensing of Environment 103:465-473.

Zerda H.R. \& Moreira M.L. 2006. Subregiones Naturales de Santiago del Estero. En: condiciones Naturales, Atlas Geográfico de Santiago del Estero. Atlas geográfico de la provincia de Santiago del Estero. Ed. Universidad

Católica de Santiago del Estero. CD ROM. ISBN 950-31-0065-8.

\footnotetext{
${ }^{1}$ INPROVE, Facultad de Ciencias Forestales - UNSE, Av. Belgrano (sur) No 1912, (CP 4200), Capital, Santiago del Estero,e-mail: mann@unse.edu.ar
} 